\title{
Impact of subsequent birth and delivery mode for women with previous OASIS: systematic review and meta-analysis
}

\author{
Sara S. Webb ${ }^{1,2}$ - Derick Yates ${ }^{2} \cdot$ Margarita Manresa $^{3}$ - Matthew Parsons ${ }^{2}$ • \\ Christine MacArthur ${ }^{1} \cdot$ Khaled M. K. Ismail $^{4}$
}

Received: 30 August 2016 / Accepted: 22 November 2016/Published online: 26 December 2016

(C) The Author(s) 2016. This article is published with open access at Springerlink.com

\begin{abstract}
Introduction and hypothesis Obstetric anal sphincter injuries (OASIS) are serious complications of vaginal birth. In a pregnancy following OASIS women may be keen to avoid an elective caesarean section, yet cautious about pursuing another vaginal birth that may result in further damage to the pelvic floor and possible long-term anal incontinence. This review aimed to evaluate the impact of subsequent birth and its mode on anal incontinence (AI) and/or quality of life (QoL), for women with previous OASIS.

Methods Searches of MEDLINE, EMBASE, CINAHL, and AMED from inception to February 2016 were undertaken with selection criteria of any study evaluating the effect of a subsequent birth on $\mathrm{AI}$ and/or QoL in women with previous OASIS. Where possible, data were extracted to populate $2 \times 2$ tables and allow meta-analysis relating to the impact of subsequent birth on $\mathrm{AI}$ and/or QoL.

Results Twenty-seven non-randomised studies were included. Meta-analysis of 14 studies (977 women) did not demonstrate any significant associations between $\mathrm{AI}$ in women with
\end{abstract}

Electronic supplementary material The online version of this article (doi:10.1007/s00192-016-3226-y) contains supplementary material, which is available to authorized users

Sara S. Webb

sara.webb@bwnft.nhs.uk

1 Institute of Applied Health Research, College of Medical and Dental Sciences, University of Birmingham, Birmingham, UK

2 Birmingham Women's NHS Foundation Trust, Delivery Suite, Mindlesohn Road, Edgbaston, Birmingham B15 2TG, UK

3 Fundació hospital, Asil de Granollers, Barcelona, Spain

4 Institute of Metabolism and Systems Research, College of Medical and Dental Sciences, University of Birmingham, Birmingham, UK previous OASIS and subsequent birth or its mode. Impact on QoL was reported in 12 studies (912 women); however, difference in outcome reporting precluded data meta-analysis. Conclusions Comparisons of outcomes and effective synthesis were limited by sample size, quality and heterogeneity of the studies included. Consequently, the optimal mode of delivery for women with previous OASIS is still not known and better data are needed.

Keywords Bowel function · OASIS · Quality of life · Subsequent birth $\cdot$ Systematic review

\section{Introduction}

Obstetric anal sphincter injuries (OASIS) are a serious complication of vaginal birth with a reported UK incidence ranging from 0 to $8 \%$ [1]. They are recognised to be a major risk factor for anal incontinence (AI), resulting in concern amongst some women considering subsequent mode-of-delivery after having sustained an OASIS. Recent UK data demonstrate a steadily rising incidence of this type of trauma over the past decade, possibly because of the increased awareness and improved methods of detection [2]. Using an average prevalence of $5 \%$, it is estimated that 30,000 women in the UK sustain OASIS annually. Even though the risk of AI is substantially increased after OASIS most women with this injury have no bowel problems [3]. For these women and in the absence of an obvious sphincter defect on ultrasound, the Royal College of Obstetricians and Gynaecologists (RCOG) recommends discussion and consideration of all modes of birth, based on limited, low level 4 evidence [3]. Indeed, data show that, before consideration of AI symptoms, over $60 \%$ of women with previous OASIS would prefer a subsequent vaginal birth $[4$, 5]. Therefore, the majority of women seem to be keen to avoid 
unnecessary major surgical intervention, such as caesarean section, although a significant number are still cautious about pursuing another vaginal birth that could result in further damage to the pelvic floor and long-term AI. This systematic review is aimed at assessing currently available evidence to guide women with previous clinically diagnosed OASIS in making an informed choice about subsequent births and mode of delivery.

\section{Materials and methods}

A protocol using widely recommended methods for systematic reviews of observational studies was developed and registered with PROSPERO International prospective register of systematic reviews $[6,7]$. The PRISMA statement and checklist were followed throughout review preparation (Appendix S1).

MEDLINE, EMBASE, CINAHL and AMED databases were searched electronically from inception to February 2016. A combination of medical subject headings (MeSHs), to encompass both bowel function and quality of life, keywords, and word variants using Boolean operators "OR" and "AND" to capture relevant text citations were used. Search strategies were adapted for each database (Appendix S2). The term "subsequent birth" was not included in the original search to reduce the risk of limiting access to all possibly relevant articles. In addition, reference lists of relevant articles were manually searched to identify papers not captured by electronic searches. The search focused on capturing any randomised controlled trials (RCTs) or nonrandomised studies (NRS) studies reporting the impact of a subsequent birth on bowel function and/or quality of life for women with previous OASIS. Case series and case reports were excluded. Conference papers and abstracts were included if they contained sufficient information regarding study design and outcome data. No language restrictions were applied, but the search was limited to human studies. The search strategies were developed by two reviewers: SSW and DY. A database of all abstracts of citations was compiled.

Studies were selected in a three-stage process. First, each title and abstract were assessed by two reviewers (SSW and $\mathrm{MM}$ ) and full articles of all references that were likely to fulfil predefined criteria were obtained. These articles were then assessed by two independent reviewers (SSW and DY), against pre-designed inclusion/exclusion criteria, with any discrepancies referred to a third party (KMI) for the final decision. Studies were included if they gave information with supporting statistical evidence on AI and/or QoL for women with previous OASIS undergoing a subsequent birth.

Data were extracted on study quality, participants' characteristics and impact of subsequent birth and mode, on bowel function, including de novo symptoms or changes in pre- existing symptoms, and/or QoL using a pre-designed data capture form. Data extraction was performed by two reviewers (SSW and MM), with assistance from a third reviewer should a discrepancy occur (DY). The primary outcome was the impact of having a subsequent birth on AI and/or QoL for women with previous OASIS. Our definition encompassed the International Continence Society (ICS)-recognised definition of AI, which is involuntary loss of flatus, liquid or solid faeces, and also faecal urgency [8]. When extracting data, it was noted whether the studies considered each of these elements in isolation or as composites. Whenever possible, data were extracted to compute $2 \times 2$ tables where women with previous OASIS had reported the impact of the subsequent birth on $\mathrm{AI}$ and/or QoL, through either questionnaires or interviews.

Risk bias and the quality of the cohort studies included were assessed by using the Joanna Briggs Institute Prevalence Critical Appraisal Tool (Table S1) [9], and case control studies were quality assessed using the NewcastleOttawa Quality Assessment Scale (Table S2) [10]. Quality assessment was then used to assess the methodological adequacies of the studies included and assist with the interpretation of meta-analysis findings and possible bias resulting from study heterogeneity.

RevMan 5.2 was used for statistical analysis [11]. A random-effects model was used because of the high likelihood of clinical and statistical heterogeneity. Meta-analysis was performed if two or more eligible studies provided comparable data. All other eligible studies were analysed descriptively. Dichotomous data are presented as summary odds ratios with 95\% confidence intervals. Continuous data are presented as standardised mean differences. Statistical heterogeneity in the meta-analysis was assessed by using the $I^{2}$ statistic. Any value $>25 \%$ was considered significant [12] and was investigated further with a sensitivity analysis of excluding studies of markedly different study design/dataset.

\section{Results}

Twenty-seven non-randomised studies (NRSs) from 9 countries were included (25 cohort; 2 case control) (Table S3), of which 14 cohort NRSs were included for quantitative synthesis by meta-analysis (Fig. 1). No RCTs or relevant systematic reviews were identified.

Study quality assessment of all the cohort and case control studies included revealed deficiencies in many methodological areas. For the cohort NRSs, no studies met all ten quality criteria (Fig. S1). Only 1 study (3.84\%) met eight criteria, the remainder fulfilled seven or less, with 15 studies $(60 \%)$ meeting $\leq 50 \%$ of the quality criteria. Neither of the two case control studies met all nine criteria (Table S4). No studies were 
Fig. 1 PRISMA 2009 flowchart

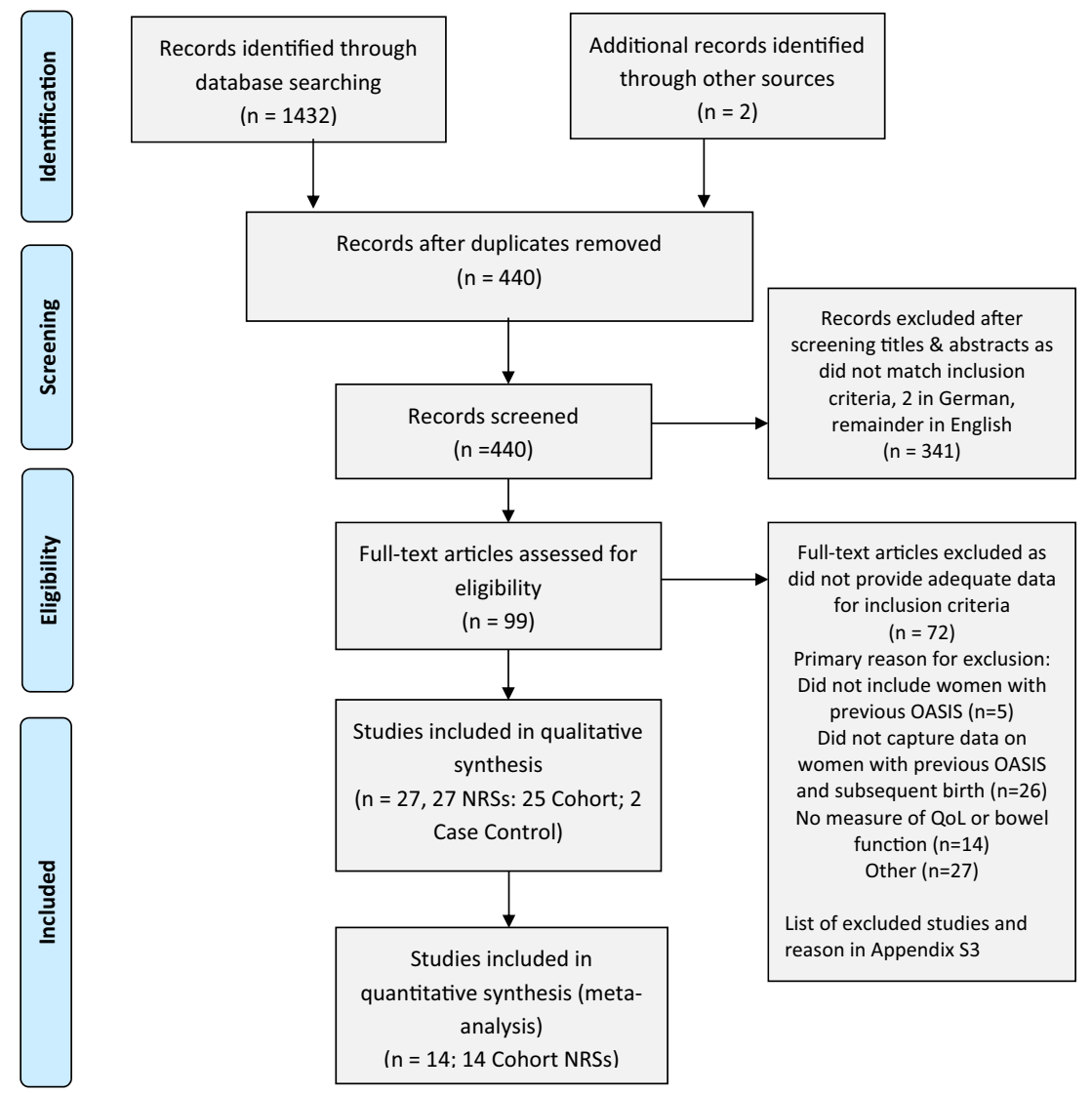

excluded from the systematic review for failure to fulfil the quality criteria.

In relation to the primary objective, 13 of the total of 27 studies which satisfied inclusion criteria, (48.2\%) were undertaken primarily to assess the impact of a subsequent birth for women with previous OASIS (Table S3). From all 27 of the studies included, a total of 3,297 women were followed up after a primary OASIS; however, data regarding the impact of subsequent birth on $\mathrm{AI}$ and/or QoL were only available for 1,781 women (54\%). Owing to the structure of the questionnaires and reporting methods for multiple symptoms, data on relevant outcomes were only available for meta-analysis on 997 (977 out of 1,$781 ; 55.9 \%$ ) of these women, in 14 studies (Table S5). Studies that could not be meta-analysed are described individually. Of the 27 studies included, 12 studies $(44.4 \%)$ considered the impact of a subsequent birth on a woman's QoL.

The use of validated measurement tools was reported in $37.1 \%$ of the studies.

Only 2 studies $(7.4 \%)[13,14]$ included details about required sample sizes to achieve adequate powering of calculations; however, these were not achieved in either study because of high attrition rates.

Fifteen $(55.5 \%)$ of the studies included used data for women who sustained and had OASIS repair before the first edition of the RCOG green top guidelines in July 2001 [3], recommending that standardised classification and repair management be introduced (Table S6).

\section{Subsequent birth vs no subsequent birth (irrespective of mode)}

\section{Impact on $A I$}

Meta-analysis of five cohort NRSs [13-17] did not demonstrate a significant difference in reported AI in women with previous OASIS who had a subsequent birth, irrespective of mode, compared with those who did not (562 women; OR 1.25; 95\% CI 0.73-2.15; $I^{2}=36 \%$; Fig. 2). Unlike all other studies included in this meta-analysis, Nordenstam et al. [17] had a primary study objective of the natural progression of AI following childbirth, not specifically for women with previous OASIS. Inclusion of this study resulted in an $I^{2}$ value of $36 \%$. We therefore repeated the meta-analysis following exclusion of this study; however, this still did not demonstrate a significant difference (532 women; OR 1.36; CI 0.84-2.19; $I^{2}=$ $25 \%$ ).

Of the two studies by Sze $[13,14]$, both undertaken in the USA, one demonstrated outcomes favouring subsequent vaginal birth for women with previous fourth degree OASIS (OR $1.88,95 \%$ CI 0.91-3.87) compared with the other, which favoured no subsequent vaginal birth for women with the 


\begin{tabular}{|c|c|c|c|c|c|c|c|c|c|c|}
\hline \multirow[b]{2}{*}{ Study or Subgroup } & \multicolumn{2}{|c|}{ No Subsequent birth } & \multicolumn{2}{|c|}{ Subsequent birth } & \multicolumn{3}{|c|}{ Odds Ratio } & \multirow{2}{*}{\multicolumn{3}{|c|}{$\begin{array}{c}\text { Odds Ratio } \\
\text { M-H, Random, } 95 \% \mathrm{Cl}\end{array}$}} \\
\hline & Events & Total & Events & Total & Weight & M-H, Random, $95 \% \mathrm{Cl}$ & Year & & & \\
\hline Kumar, 2012 & 9 & 16 & 6 & 19 & $12.0 \%$ & $2.79[0.70,11.10]$ & 2004 & 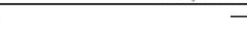 & $x_{1}$ & \\
\hline Nordenstam et al, 2009 & 1 & 4 & 16 & 26 & $4.7 \%$ & $0.21[0.02,2.29]$ & 1995 & & & \\
\hline Sze, 2005 & 11 & 65 & 23 & $10 ?$ & $25.6 \%$ & $0.74[0.34,1.65]$ & 1984 & $\longrightarrow$ & - & \\
\hline Sze, 2005 (online 2004) & 20 & 52 & 24 & 96 & $28.3 \%$ & $1.88[0.91,3.87]$ & 1984 & & $=$ & \\
\hline Sangalli et al, 2000 & 18 & 63 & 27 & 114 & $29.4 \%$ & $1.29[0.64,2.59]$ & 1982 & & & \\
\hline Total $(95 \% \mathrm{Cl})$ & & 200 & & 362 & $100.0 \%$ & $1.25[0.73,2.15]$ & & & & \\
\hline Total events & 59 & & 96 & & & & & & & \\
\hline \multicolumn{8}{|c|}{ Heterogeneity $\operatorname{Tau}^{2}=0.13 ; \mathrm{Chi}^{2}=6.28, \mathrm{df}=4(\mathrm{P}=0.18) ; \mathrm{I}^{2}=36 \%$} & \begin{tabular}{|lcl}
0.01 & 0.1 \\
& Favours no subt birth
\end{tabular} & Favours su & $\begin{array}{ll}1 \\
10 \\
\text { ubt birth }\end{array}$ \\
\hline
\end{tabular}

Fig. 2 Reported incidence of anal incontinence (AI) in women with previous obstetric anal sphincter injuries (OASIS): no subsequent birth versus subsequent birth

lower category (3c) OASIS; however, neither reached statistical significance (OR 0.74, 95\% CI 0.34-1.65).

Several studies that reported data relevant to this comparison, but could not be included in the meta-analysis provided contradictory information. In a retrospective cohort study of 125 women with matched controls, De Leeuw et al. [18] reported that for women with previous OASIS there was no association between $\mathrm{AI}$ and having a subsequent vaginal birth or not ( $41 \%$ vs $39 \%$ respectively; OR 2.32 ; $95 \%$ CI 0.85 $6.33 ; p=0.10$ ). A retrospective follow-up NRS (mean 27.5 years \pm 2.4 ) of 99 women with OASIS from their first birth by Huebner et al. [4] also found no association between parity irrespective of mode and anal incontinence of either liquid/solid stool (OR 1.69; 95\% CI $0.58-4.97 ; p=0.335$ ) or flatus (OR 2.25; 95\% CI 0.94-5.41; $p=0.067$ ). Likewise, in their study of women with OASIS and matched controls (mean follow-up 22.2 years), Soerensen et al. [19] found no association between long-term $\mathrm{AI}$ and having a subsequent birth in women with third- or fourth-degree OASIS. A retrospective cohort study by Sangalli et al. [16] reported that subsequent vaginal birth in women who previously sustained third-degree OASIS $(n=80)$ was associated with a significant decrease in the severity of AI $(p=0.02)$, whereas for women with previous fourth-degree OASIS $(n=34)$, subsequent vaginal birth was associated with an increased risk of severe incontinence $(p=0.042)$. A similar study by Bek and Lauberg [20] found a significant association between transient AI in women directly after sustaining primary "complete" OASIS and permanent $\mathrm{AI}$ after a subsequent vaginal birth (OR 8.7; 95\% CI 1.9-39; $p=0.05$ ); however, the study sample size was small $(n=56)$. Reid et al. [21] also found that having a subsequent birth was significantly associated with symptoms of $\mathrm{AI}$ at 3 years following primary OASIS $(p=0.012)$. Similarly, in a small study $(N=117)$, Poen et al. [22] demonstrated a significantly higher incidence of reported symptoms of $\mathrm{AI}$ in women with subsequent birth versus those without (RR 1.6; 95\% CI $1.1-2.5 ; p=0.025$; mean follow-up period was 4.7 years; range $0.8-11.3$ ). Visscher et al. [23] found that AI was increased in women with subsequent birth relative to those without $(p=0.008)$, but this was a very small study that excluded all women who were asymptomatic following their first OASIS.

Three studies provided data on AI symptoms in relation to the total number of subsequent births following OASIS [13, 14, 17]. Meta-analysis of these did not demonstrate a difference in reported AI related to one compared with two or more subsequent vaginal births for any category of OASIS (two studies, 210 women; OR 0.88; 95\% CI $0.40-1.94 ; I^{2}=19 \%$; Figure S2), or for women with a previous fourth-degree OASIS (two studies, 130 women; OR $0.94 ; 95 \%$ CI $0.39-2.31 ; I^{2}=12 \%$; Figure S2).

Regarding a change in the reported AI, meta-analysis of eight cohort NRSs [17, 20, 24-29], demonstrated that there was no significant change in reported AI symptoms in women with previous OASIS before and following their subsequent birth, irrespective of mode (438 women; OR 1.04; $95 \%$ CI $0.58-1.85 ; I^{2}=34 \%$; Fig. 3). Unlike all other studies included in the meta-analysis, Tetzschner et al. [29] and Bondili et al. [25] reported findings in women with subsequent elective caesarean section and inclusion of these two studies in the meta-analysis resulted in an $I^{2}$ value of $34 \%$. A repeat metaanalysis without the inclusion of these studies still did not demonstrate a significant worsening in AI for women with previous OASIS following a subsequent vaginal birth (131 women; OR of 1.36 ; CI $0.72-2.59 ; I^{2}=0 \%$ ).

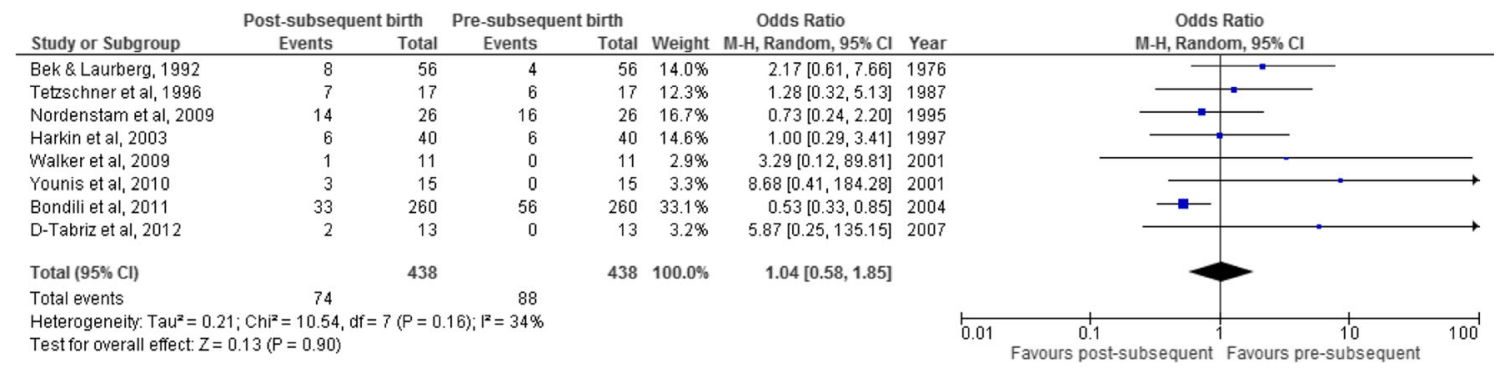

Fig. 3 Reported incidence of AI in women with previous OASIS: pre- versus post-subsequent birth 
With regard to individual studies, An et al. [31] showed that in her sample of 67 women with previous OASIS, $82 \%$ reported AI symptoms to be the same or improved following a subsequent birth and concluded that low AI measurement scores pre-subsequent birth were a significant predictor of normal continence post-subsequent birth $(p=0.0002)$.

\section{Impact on $Q o L$}

Quality of life was studied in only one small case control study of women who sustained recurrent OASIS in their subsequent birth (cases $n=34$ ) compared with women who did not, matched for age and ethnicity (controls $n=34$ ), showing no change to QoL for women at 12 weeks postpartum compared with antenatal parameters, nor between the two groups [31].

\section{Vaginal birth vs caesarean section}

\section{Impact on $A I$}

Three cohort NRSs were meta-analysed for mode of subsequent birth [29, 31, 32], which did not demonstrate any difference in de novo AI or worsening of symptoms in women with previous OASIS following subsequent vaginal birth relative to subsequent caesarean section (three studies, 195 women; OR $0.61 ; 95 \%$ CI $0.20-1.90 ; I^{2}=0 \%$; Fig. 4).

Similar to other outcomes, individual studies produced mixed findings. In a prospective cohort study after primary sphincter repair, Reid et al. [21], found that at 3-year follow-up, AI symptoms were more frequent in women with subsequent caesarean section (5 out of 92); however, they attributed this to the fact that symptomatic women were offered elective caesarean section.

Naidu et al. [33], Fitzpatrick et al. [34], and Jorden et al. [31], found no worsening of AI symptoms for women having whichever mode of subsequent birth they were recommended by their clinician. Scheer et al. [35], using a validated questionnaire, demonstrated an improvement in all symptoms of AI except solid incontinence, after subsequent vaginal birth; however, again, the study only included women who underwent their recommended mode of subsequent birth and was very small $(n=35)$.

\section{Impact on $Q o L$}

Scheer et al. [35] also studied QoL and found a significant negative impact on three domains post-birth: incontinence impact $(p=0.012)$, emotions $(p=0.003)$, and severity measures $(p=0.032)$, for women $(n=9)$ having subsequent recommended caesarean section (because of the substantial compromised anal function), compared with those undergoing a recommended vaginal birth.

\section{Discussion}

This systematic review summarises the available evidence regarding the impact of subsequent birth for women with a previous history of OASIS on AI and/or QoL. As no RCTs were identified, this is based on data from 27 cohort and case control NRSs, across nine countries, predominantly with methodological inadequacies (data provided for 1,781 of the 3,297 women where data relating to subsequent births following OASIS were available) [4, 13-33, 35-41].

Meta-analysis did not demonstrate a difference in AI in women with previous OASIS who had a subsequent birth compared with those who did not (five studies; 562 women); or a change in AI in women with previous OASIS before and following subsequent birth irrespective of mode (eight studies; 438 women); or a difference in de novo AI or worsening symptoms in women with previous OASIS following subsequent vaginal birth compared with subsequent caesarean section (four studies; 211 women).

Despite QoL being an important indicator for women with previous OASIS when deciding on future pregnancy and birth mode, research in this area was limited (12 studies, 912 women) and no data were suitable for meta-analysis owing to differences in outcome reporting between studies.

The several strengths to this systematic review include rigorous searching, study selection, quality appraisal and data extraction methodology. The term "subsequent birth" was not included in the original search to reduce the risk of limiting access to all possibly relevant articles. Also, not restricting NRSs enabled all possible studies to be included.

The main limitation of the review findings arises from both the quality and heterogeneity of the individual studies on which they are based, with the majority of studies not looking

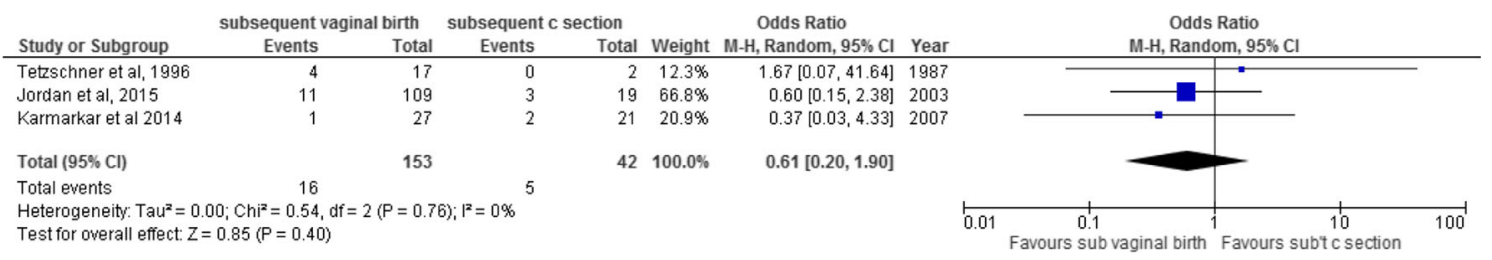

Fig. 4 Incidence of worsening of or de novo symptoms of AI in women with previous OASIS: subsequent vaginal birth versus subsequent caesarean section 
at symptom severity and/or small sample sizes. All studies satisfying the inclusion criteria were NRSs, 14 (52\%) not conducted with the primary intention of investigating the impact of a subsequent birth on AI and/or QoL for women with previous OASIS. Only 14 of the studies included reported data allowing inclusion in meta-analyses. Consequently, the risk that findings from a meta-analysis of NRSs are subject to over-exaggeration of the tested intervention (i.e., subsequent birth or its mode) because of methodological biases must be acknowledged [42]. However, lack of difference found from meta-analyses undertaken mitigates this potential risk. Data on confounding variables were also limited in many of the studies and must be taken into account in future research. Another consideration is improvement in OASIS recognition and repair. Structured training, use of recommended suture materials and repair techniques are associated with good clinical outcomes [36, 43, 44]. Attention to the above was driven by the RCOG Green-top guideline first published in 2001 [3]. Consequently, data from women delivered before these recommendations (55.5\% of the NRSs included) may not be representative of those in centres where recommended interventions for OASIS assessment and repair have been implemented. A sub-analysis of studies with data from women having a subsequent birth after 2003 (to allow time for RCOG evidence-based recommendations to become embedded in clinical practice), limited the number of studies eligible for inclusion and when meta-analysis was still possible, did not show any differences in findings (Appendix S4). However, some of these results should be interpreted with caution because of evidence of significant statistical heterogeneity (Appendix S4 Fig. S4b). Nevertheless, we believe that, because of a lack of evidence of the universal adoption of this practice and increasing mobility of women between units and countries, the results of this review remain relevant. Further sub-analysis to assess the impact of suture material, repair method, follow-up on clinical outcomes would have complimented this review; however, data are not readily available in the studies included.

Recognition and primary repair of OASIS immediately following birth has improved [2]; moreover, sustaining OASIS has not been demonstrated as a factor deterring women from having subsequent pregnancies [45]. The main focus for clinicians is helping women to choose the optimal mode of subsequent birth. In our clinical experience, there is a wide variation between individual women with regard to their choice of mode of subsequent birth. Some are prepared to pursue another vaginal birth, despite evidence suggesting that the risk of an OASIS in a subsequent vaginal birth might be greater than for women with no previous history of OASIS [4], although other women request a caesarean section irrespective of health practitioner advice. Interestingly, the study by Bondili et al. [25], found improvements in $\mathrm{AI}$ in symptomatic women recommended to undergo subsequent elective caesarean section.
This could be improvement influenced by achieving the desired mode of birth, learning to cope with/adapt to symptoms of $\mathrm{AI}$ in the longer term, or actual improvement because of management interventions such as dietary changes or physiotherapy. It is important to highlight the difference in the follow-up period between these two studies -6 months and 10 years respectively. Although these findings remain a matter of debate, they demonstrate the psychological complexity of pregnancy and giving birth and that OASIS and its long-term complications cannot be considered in isolation. It is therefore interesting that this review highlights that over half of the research suitable for inclusion concentrates on the occurrence of AI for women with OASIS undergoing a subsequent pregnancy and birth, but not its severity or impact on QoL.

Women may wish to pursue their desired mode of subsequent birth; however, pregnancy and childbirth is a dynamic process with unpredictable events necessitating unplanned interventions. The majority of studies excluded women who did not obtain their planned mode of subsequent birth (through maternal choice or clinical need), or had a subsequent caesarean section. This affects representativeness, as AI may be a consequence of other factors related to pregnancy and labour, such as pudendal neuropathy, prolonged labour, instrumental delivery, or even pregnancy itself.

The current RCOG guideline [3] acknowledges that the level of evidence supporting their recommendations regarding the mode of subsequent birth for women with previous OASIS is low (level 4). However, in our opinion, this review clearly demonstrates that current evidence is substantially limited to provide any meaningful guidance. It also highlights, as there are few studies involving women assessed and repaired using 2001 RCOG recommendations, that there is currently no literature reporting long-term outcomes of bowel function and quality of life for these women who undergo a subsequent birth. This calls for urgent collaborative prospective work to generate the evidence required to inform practice.

\section{Conclusion}

In the absence of higher quality evidence this systematic review and meta-analysis supports the current recommendation of a subsequent vaginal birth for women with previous OASIS who demonstrate no AI symptoms or sphincter defects. However, evidence is urgently needed to support or refute the practice of recommending elective caesarean section for symptomatic women or those with ultrasound anal sphincter abnormalities.

Findings from this review support the RCOG guideline [3] recommendation for further research. If an RCT to assess the impact of mode of subsequent birth following OASIS on both AI and QoL was deemed acceptable by women, such a trial will need to be multicentre or indeed international to ensure 
timely conclusion without compromising its power to address important outcomes. A more immediate option would be a well-conducted, appropriately sized prospective cohort study of women with previous OASIS undergoing subsequent birth, with primary objectives of assessment of anal function, QoL and sphincter anatomy both before and after the intervention with on-going follow-up.

\section{Compliance with ethical standards}

Details of ethics approval No ethical approval required.

Funding SSW is partly funded by a National Institute for Health Research (NIHR) clinical doctoral fellowship (CDRF-2012-03-064). CM is part funded by the NIHR CLAHRC-WM programme. This article/paper/report presents independent research funded by the National Institute for Health Research (NIHR). The views expressed are those of the author(s) and not necessarily those of the NHS, the NIHR or the Department of Health.

\section{Conflicts of interest None.}

Open Access This article is distributed under the terms of the Creative Commons Attribution 4.0 International License (http:// creativecommons.org/licenses/by/4.0/), which permits unrestricted use, distribution, and reproduction in any medium, provided you give appropriate credit to the original author(s) and the source, provide a link to the Creative Commons license, and indicate if changes were made.

\section{References}

1. Thiagamoorthy G, Johnson A, Thakar R, Sultan A. National audit to assess the true incidence of perineal trauma and its subsequent management in the United Kingdom. BJOG. 2013;120:478-9.

2. Gurol-Urganci ICD, Edozien LC, Mahmood TA, Adams EJ, Richmond DH, Templeton A, et al. Third- and fourth-degree perineal tears among primiparous women in England between 2000 and 2012: time trends and risk factors. BJOG. 2013;120:1516-25.

3. RCOG. Third- and Fourth-degree Perineal Tears, Management (Green-top Guideline 29). RCOG Greentop Guidelines. London: RCOG ;2015.

4. Huebner M, Gramlich NK, Rothmund R, Nappi L, Abele H, Becker $\mathrm{S}$. Fecal incontinence after obstetric anal sphincter injuries. Int $\mathrm{J}$ Gynecol Obstet. 2013;121(1):74-7.

5. Faltin D, Petignat P, Reuse C, Drumps P, Nunno C, Pierret B, et al. A prospective cohort study of vaginal delivery after a previous anal sphincter tear. Neurourol Urodyn. 2005;24:5-6.

6. Stroup D, Berlin J, Morton S, Olkin I, Williamson G, Rennie D, et al. Meta-analysis of observational studies in epidemiology. JAMA. 2000;283:2008-12.

7. Webb S, Yates D, Ismail K. The impact of subsequent birth on women with previous Obstetric Sphincter Injury (OASIS) on Quality of Life (QoL) and bowel function: a systematic review. PROSPERO 2014:CRD420140092102014. Available from: http://www.crd.york.ac.uk/PROSPERO_REBRANDING/display_ record.asp?ID=CRD42014009210.

8. Norton C, Christiansen J, Butler U, Harai D, Nelson R, Pemberton J, et al. Anal incontinence. In: Ambrams P, Cardozo L, Khoury S, Wein A, editors. Incontinence: second international consultation on incontinence. Plymouth: Health Books; 2002.
9. Munn Z, Moola S, Riitano D, Lisy K. The development of a critical appraisal tool for use in systematic reviews addressing questions in prevalence. Int J Health Policy Manag. 2014;3(3):123-8.

10. Wells G, Shea B, O'Connell D, Peterson J, Welch V, Losos M, et al. The Newcastle-Ottawa Scale (NOS) for assessing the quality of nonrandomized studies in meta-analyses. 2009. Available from: http://www.ohri.ca/programs/clinical_epidemiology/oxford.htm.

11. Review Manager (REVMAN) [Computer programme] Version 5.2. Copenhagen. The Nordic Cochrane Centre: The Cochrane Collaboration, 2012.

12. Higgins J, Thompson S, Deeks J, Altman D. Measuring inconsistency in meta-analyses. Br Med J. 2003;327:557-60.

13. Sze EH. Prevalence and severity of anal incontinence in women with and without additional vaginal deliveries after a fourthdegree perineal laceration. Dis Colon Rectum. 2005;48(1):66-9.

14. Sze EH. Anal incontinence among women with one versus two complete third-degree perineal lacerations. Int J Gynaecol Obstet. 2005;90(3):213-7.

15. Kumar R. Anal incontinence and quality of life following obstetric anal sphincter injury. Arch Gynecol Obstet. 2012;285(3):591-7.

16. Sangalli MR, Floris L, Faltin D, Weil A. Anal incontinence in women with third or fourth degree perineal tears and subsequent vaginal deliveries. Aust N Z J Obstet Gynaecol. 2000;40(3):244-8.

17. Nordenstam J, Altman D, Brismar S, Zetterstrom J. Natural progression of anal incontinence after childbirth. Int Urogynecol J. 2009;20:1029-35.

18. De Leeuw JW, Vierhout ME, Struijk PC, Hop WC, Wallenburg HC. Anal sphincter damage after vaginal delivery: functional outcome and risk factors for fecal incontinence. Acta Obstet Gynecol Scand. 2001;80(9):830-4.

19. Soerensen MM, Buntzen S, Bek KM, Laurberg S. Complete obstetric anal sphincter tear and risk of long-term fecal incontinence: a cohort study. Dis Colon Rectum. 2013;56(8):992-1001.

20. Bek KM, Laurberg S. Risks of anal incontinence from subsequent vaginal delivery after a complete obstetric anal sphincter tear. Br J Obstet Gynaecol. 1992;99(9):724-6.

21. Reid A, Beggs A, Sultan A, Roos A, Thacker R. Outcome of repair of obstetric anal sphincter injuries after three years. Int J Gynaecol Obstet. 2014;127(1):47-50.

22. Poen AC, Felt-Bersma RJ, Strijers RL, Dekker GA, Cuesta MA, Meuwissen SG. Third-degree obstetric perineal tear: long-term clinical and functional results after primary repair. Br J Surg. 1998;85(10):1433-8.

23. Visscher AP, Lam TJ, Hart NA, Mulder CJ, Felt-Bersma RJ. Anal incontinence, sexual complaints, and anorectal function in patients with a third degree anal sphincter rupture: Long term follow-up. Gastroenterology. 2013;144(5 Suppl 1):368.

24. Dilmaghani-Tabriz D, Soliman N. Delivery after third-or fourthdegree perineal tear. BJOG. 2012;119.

25. Bondili A, Siddiqui I, Nichols W, Kettle C, Cooper J, O'Mahony F, et al. Management of pregnancies following third and fourth degree perineal tears: a multi disciplinary approach. Int Urogynecol J Pelvic Floor Dysfunct. 2011;22: S120-1.

26. Walker HJ, Gelman W, Thomas JM, Bearn PE, McWilliams D. Does vaginal delivery after Obstetric Anal Sphincter Injury (OASI) cause further damage? Int Urogynecol J Pelvic Floor Dysfunct. 2009;20(3 Suppl):S278-9.

27. Younis J, Rajendran N, Walker H, Wolf G, McWilliams D, Bearn P. Deterioration of anal sphincter function following second vaginal delivery in women with previous intrapartum perineal tears. Colorectal Dis. 2010;12.

28. Harkin R, Fitzpatrick M, O'Connell PR, O’Herlihy C. Anal sphincter disruption at vaginal delivery: is recurrence predictable? Eur J Obstet Gynecol Reprod Biol. 2003;109(2):149-52. 
29. Tetzschner T, Sorensen M, Lose G, Christiansen J. Anal and urinary incontinence in women with obstetric anal sphincter rupture. $\mathrm{Br} \mathrm{J}$ Obstet Gynaecol. 1996;103(10):1034-40.

30. An V, D'Souza B, Thomas E, Schierlitz L, Woods R, Keck J. Obstetric anal sphincter injuries; outcome and function after subsequent deliveries. Tripartite Colorectal Meeting of the American Society of Colon and Rectal Surgeons, ASCRS; Association of Coloproctology of GB and Ireland; the Section of Coloproctology, Royal Society of Medicine; the Colon and Rectal Surgery Section, Royal Australasian Birmingham United Kingdom: Conference Publication (var. pagings). 2014; 16:89.

31. Jordan P, Naidu M, Sultan A, Thacker R. Effect of subsequent vaginal delivery on bowel symptoms and anorectal function in women who sustained a previous obstetric anal sphincter injury (OASI). International Urogynaecology Journal and Pelvic Floor Dysfunction 40th Annual Meeting of the International Urogynecological Association, IUGA 2015; Nice, France: Conference Publication (var. pagings). 2015;26(1 Suppl 1):S40-1.

32. Karmarkar R, Bhide A, Digesu A, Khullar V, Fernando R. Mode of delivery after previous obstetric anal sphincter injuries (OASIS). Eur J Obstet Gynecol Reprod Biol. 2015;194:7-10.

33. Naidu M, Sultan A, Thakar R. Outcome of anal symptoms and anorectal function following two obstetric anal sphincter injuries (OASIS) - a prospective case controlled study. International Urogynaecology Journal and Pelvic Floor Dysfunction 40th Annual Meeting of the International Urogynecological Association, IUGA 2015; Nice, France 2015. p. S40-S1.

34. Fitzpatrick M, Cassidy M, Barussaud M, Hehir M, O'Herlihy C. Does anal sphincter injury preclude subsequent vaginal delivery? Eur J Obstet Gynecol Reprod Biol. 2016;198:30-4.

35. Scheer I, Thakar R, Sultan AH. Mode of delivery after previous obstetric anal sphincter injuries (OASIS)-a reappraisal? Int Urogynecol J Pelvic Floor Dysfunct. 2009;20(9):1095-101.
36. Andrews V, Shelmeridine S, Sultan AH, Thakar R. Anal and urinary incontinence 4 years after a vaginal delivery. Int Urogynecol J Pelvic Floor Dysfunct. 2013;24(1):55-60.

37. Daly JO, Sultan AH, Van KW, Thakar R. Outcome of childbirth after previous obstetric anal sphincter injury. Int Urogynecol J Pelvic Floor Dysfunct. 2013;24:S69-70.

38. Faltin DL, Sangalli MR, Roche B, Floris L, Boulvain M, Weil A. Does a second delivery increase the risk of anal incontinence? BJOG. 2001;108(7):684-8.

39. Samarasekera DN, Bekhit MT, Wright Y, Lowndes RH, Stanley $\mathrm{KP}$, Preston JP, et al. Long-term anal continence and quality of life following postpartum anal sphincter injury. Colorectal Dis. 2008;10(8):793-9.

40. Wagenius J, Laurin J. Clinical symptoms after anal sphincter rupture: a retrospective study. Acta Obstet Gynecol Scand. 2003;82(3): 246-50.

41. Yousif S, Eogan M. Mode of delivery after previous anal sphincter injury (ASI): role of the perineal clinic. Ir J Med Sci. $2011 ; 180$.

42. Reeves B, Deeks J, Higgins J, Wells G. Chapter 13: Including nonrandomised studies. In: Higgins J, Green S, editors. Cochrane Handbook for Systematic Reviews of Interventions Version 5-0-1. www.cochrane-handbook.org: The Cochrane Collaboration 2008.

43. Fernando RJ, Sultan AHH, Kettle C, Thakar R. Methods of repair for obstetric anal sphincter injury. Cochrane Database Syst Rev. $2013 ; 12$.

44. Williams A, Adams E, Tincello D, Alfirevic Z, Wakinshaw S, Richmond $\mathrm{D}$. How to repair and anal sphincter injury after vaginal delivery: results of a randomised controlled trial. BJOG. 2006;113: 201-7.

45. Baghestan E, Irgens L, Rasmussen S. Risk of recurrence and subsequent delivery after obstetric anal sphincter injuries. BJOG. 2012;119(1):62-9. 